\title{
Right hepatic artery pseudoaneurysm: rare complication of plastic biliary stent insertion
}

A 68-year-old-woman was referred to our hospital because of obstructive jaundice. The patient had itching, but no abdominal pain, fever, or weight loss. Laboratory tests showed elevated levels of alkaline phosphatase (2035 U/L; normal 35-104U/L), r-glutamyltransferase (497 U/L; normal $<39 \mathrm{U} / \mathrm{L})$, and bilirubin $(16.8 \mathrm{mg} / \mathrm{dL}$; normal $<1.2 \mathrm{mg} / \mathrm{dL}$ ). C-reactive protein $(1.35 \mathrm{mg} / \mathrm{L}$; normal $<0.5 \mathrm{mg} / \mathrm{L})$ and tumor antigen CA 19-9 (960.8 U/L; normal $<37 \mathrm{U} / \mathrm{L}$ ) levels were slightly raised. Abdominal computed tomography (CT) revealed enhanced wall thickening of the intrahepatic duct (IHD), the confluent portion, and the common hepatic duct (CHD), which was compatible with Klatskin's tumor, bismuth type IIIa. CT also revealed massive tumor invasion of the right hepatic artery ( Fig. 1). We performed emergency endoscopic retrograde cholangiopancreatography (ERCP) and a plastic biliary stent $(8.5 \mathrm{Fr}, 10 \mathrm{~cm})$ was inserted into the right hepatic bile duct. Cytological examination of the bile revealed a class $\mathrm{V}$ adenocarcinoma. After 20 days, the patient again had fever, jaundice, and tarry stool. We carried out a repeat emergency ERCP and endoscopic nasobiliary drainage (ENBD). The patient was diagnosed as having hemobilia, since the ERCP showed blood oozing from the papilla of Vater. Enhanced $\mathrm{CT}$ revealed a hepatic artery pseudoaneurysm compressing the bile duct in the right lobe ( Fig. 2 ). We carried out hepatic angiography and coil embolization without any complications. Following this procedure there were no further episodes of hemobilia. When blood was no longer detected in the ENBD tube, endoscopic placement of bilateral metallic stents was carried out for malignant hilar biliary obstruction ( $\$$ Fig. 3 ). The patient was then given systemic chemotherapy with gemcitabine. At 10 months' followup, she remained in good condition, without clinical or laboratory evidence of hemobilia.

Hepatic artery pseudoaneurysms are rare vascular lesions. One case of hemobilia after plastic biliary stenting has been reported previously [1]. In the present case, we suspect that the biliary stent was the cause because a hepatic artery pseudoaneurysm was being compressed by the stent. However, tumor invasion and cholangitis may also contribute to the formation of an aneurysm [2]. Angiography has replaced surgery as the most useful diagnostic and therapeutic modality for hepatic artery pseudoaneurysms [3]. This report describes a hepatic artery pseudoaneurysm occurring as a rare complication of plastic biliary stent insertion and successfully treated with coil embolization.

Endoscopy_UCTN_Code_CPL_1AK_2AD

\section{Competing interests: None}

H. Inoue ${ }^{1}$, S. Tano ${ }^{1}$, R. Takayama ${ }^{1}$, K. Nimomiya ${ }^{1}$, K. Nishikawa ${ }^{1}$, M. Katurahara $^{2}$, N. Horiki ${ }^{2}$, Y. Takei ${ }^{1}$

Department of Gastroenterology and Hepatology, Mie University Graduate School of Medicine, Mie, Japan

2 Department of Endoscopic Medicine, Mie University Graduate School of Medicine, Mie, Japan

\section{References}

1 Wolters F, Ryan B, Beets-Tan R et al. Delayed massive hemobilia after biliary stenting. Endoscopy 2003; 11: 976-977

2 Monroe PS, Deeter WT, Rizk P. Delayed hemobilia secondary to expandable metal stent. Gastrointest Endosc 1993; 39: 190 191

3 Green MHA, Duell RH, Johnson CD et al. Haemobilia. Br J Surg 2001; 88: 773 -786

\section{Bibliography}

DOI $10.1055 / \mathrm{s}-0030-1256942$

Endoscopy 2011; 43: E396

(c) Georg Thieme Verlag KG Stuttgart · New York . ISSN 0013-726X

\section{Corresponding author}

\section{H. Inoue}

Department of Gastroenterology and Hepatology Mie University Graduate School of Medicine 2-174 Edobashi Tsu

Mie

Japan

Fax: +81-059-232-5223

hiro1024@clin.medic.mie-u.ac.jp

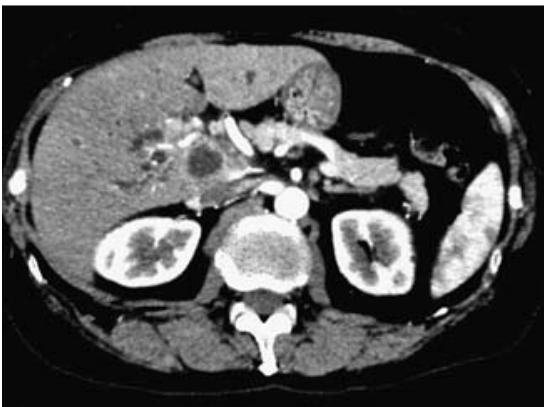

Fig. 1 Computed tomography (CT) scan showing massive tumor invasion of the right hepatic artery, in a 68-year-old woman with obstructive jaundice.

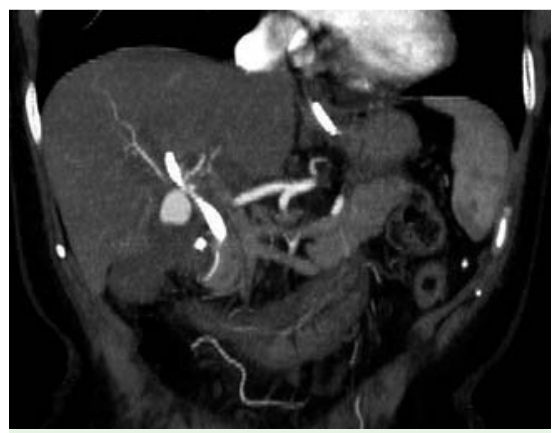

Fig. 2 Enhanced computed tomography (CT) scan showing a hepatic artery pseudoaneurysm compressing the bile duct in the right lobe.

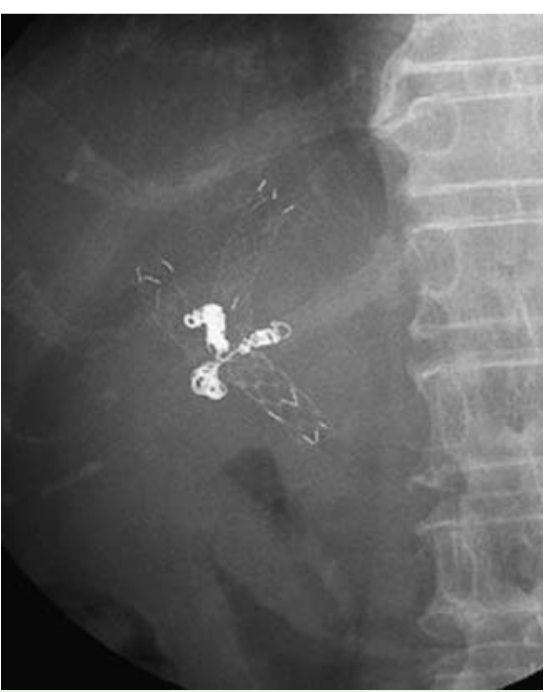

Fig. 3 After coil embolization, endoscopic placement of bilateral metallic stents was successfully carried out. 